\title{
TRANSIENT THERMOELASTIC CONTACT PROBLEM OF TWO SLIDING HALF-PLANES*
}

\author{
A. AZARKHIN and J. R. BARBER
}

Department of Mechanical Engineering and Applied Mechanics, University of Michigan, Ann Arbor, MI 48104 (U.S.A.)

(Received June 19, 1984; accepted September 17, 1984)

\section{Summary}

A transient contact problem with frictional heating for two sliding halfplanes is considered. One of the half-planes is slightly rounded to give a hertzian initial pressure distribution; the other is a rigid non-conductor. It is shown that if the ratio of initial width of contact to the width in the steady state is less than some critical value, the contact area shrinks smoothly until the steady state is reached. Otherwise the pressure distribution develops a wavy perturbation and eventually bifurcates. Results are compared with previous approximate solutions.

\section{Introduction}

Let us consider two sliding solids which are nominally conforming over some contact area. An asperity or another imperfection disturbs the pressure distribution and hence the heat generation due to friction. This produces non-uniform thermoelastic displacements in the solids and further nonuniformity in the pressure distribution. This phenomenon, which is known as thermoelastic instability, was observed experimentally by Parker and Marshall [1] and explained in terms of thermoelastic displacements in refs. 2 and 3 . Extensive study of the process has been conducted by the research group of R. A. Burton. In particular, they have shown [4 - 6] that for the two-dimensional geometry there exists some critical value $V_{c x}$ of speed for which the pressure, if started with a sinusoidal disturbance, remains unchanged. The critical speed depends on the wavelength of the disturbance. If the given velocity $V$ exceeds the critical value, the pressure grows without limits, and otherwise it eventually decays. It was assumed $[4,6]$ that contact between the two solids is maintained everywhere along the sliding surfaces by a superposed uniform pressure. If the superposed pressure is not sufficient (which is sooner or later always the case for $V>V_{\mathrm{cr}}$ ) a patch-like contact eventually develops.

*Paper presented at the Third Workshop on Thermomechanical Effects in Sliding Systems, Hanover, NH, U.S.A., June $18-20,1984$. 
Steady state solutions for patch-like contact are given in refs. 7 - 9 . There is a gap between these two problems which can be formulated as follows. Let us suppose we started with the two solids conforming initially everywhere. Let us suppose also that the force, pressing one of the bodies against the other, and the spced of sliding are given. How, generally speaking, does the process develop with time? In particular, does the contact area shrink smoothly until the steady state solution is reached or does it bifurcate? A simplified model for the axisymmetric non-steady problem has been suggested in ref. 10 where the thermally distorted body is approximated by a quadratic surface. This model has been used to evaluate the effect of design and operating conditions on the maximum temperature reached in brakes [11]. Among other things, it was shown that, in the case of uniform deceleration, the duration of the stop is significant. If the stop is sufficiently slow for hot spots to develop, the temperature is high. High temperature is also reached if the stop is sufficiently fast, owing to the high rate of heat generation. There exists an optimum between these two extremes. One of the purposes of the present paper is to determine the range of validity of this approximate treatment.

\section{The model}

To make the problem more tractable, and yet not without significance, it will be subjected to the following restrictions (Fig. 1).

(1) The contact area is stationary with respect to one solid.

(2) The other solid is a rigid non-conductor.

(3) There is no coupling between tangential and normal tractions.

(4) The conducting body is slightly rounded to give the initial pressure distribution.

Some remarks should be made about these assumptions. Assumption (1) is not independent and follows from the others. For the steady problem, roundedness of the conductor is not required for the area of contact to be stationary with respect to the conductor [6].
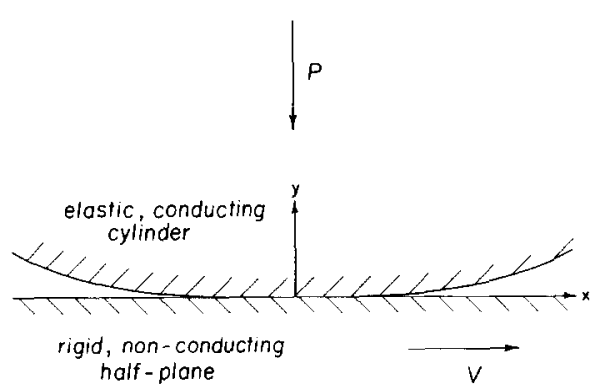

Fig. 1. The model of the surface contact. 
Rigidity of the non-conductor is not restrictive because its contribution to the elastic displacements can be absorbed in the displacements of the other body by an appropriate choice of the elastic constants. As remarked by Burton et al. [8], taking $K=0$ for one body results in the worst possible case for instability to develop. It is a reasonable approximation for a low conductivity composition brake material sliding on steel.

Assumption (3) does not mean that the tangential traction on the surface is neglected. Indeed, the work done against these tractions is the source of the heat generation. However, the elastic displacements normal to the surface, caused by the tangential tractions, are much smaller than those produced by the normal tractions, and the coupling effect is negligible. This approximation becomes exact if Dundurs' constant $\beta$ is zero [12].

\section{The method of solution and governing equations}

A straightforward method of treating the problem is to write down the governing integral equations in terms of appropriate Green's functions [13]. To set up these equations we use the fundamental solution corresponding to the release at time $t=0$ of a quantity of heat $Q$ per unit length in the $z$ direction at $x=y=0$ on the surface of the half-plane $y>0$. The temperature $T$ is given by [14]

$$
T=\frac{Q \exp \left(-R^{2}\right)}{2 \pi k \rho c t}
$$

where

$$
R^{2}=\frac{x^{2}+y^{2}}{4 k t}
$$

The corresponding normal displacements on the traction-free surface are [15]

$u_{y}=-\frac{\alpha Q(1+\nu)}{\pi \rho c(k t)^{1 / 2}} \phi_{1}(R)$

In eqns. (1) and (3), $\alpha, k, \rho, c$ and $\nu$ are respectively the coefficient of thermal expansion, the thermal diffusivity, the density, the specific heat and Poisson's ratio for the material. The function

$\phi_{1}(R)=\frac{2}{\pi^{1 / 2}} \frac{\exp \left(-R^{2}\right)}{R} \int_{0}^{R} \exp \left(s^{2}\right) \mathrm{d} s$

is related to the complex error function discussed by Miller and Gordon [16] (the series for small and large arguments for $\phi_{1}$ are given in ref. 15).

Using these results, the governing equation for the unknown contact pressure distribution $p(x)$ can be written 


$$
\begin{aligned}
u_{y}(x, t)= & \frac{2\left(1-\nu^{2}\right)}{\pi E} \int_{A(t)} p\left(x_{1}, t\right) \log \left|x_{1}-x\right| \mathrm{d} x_{1}- \\
& -\frac{\alpha(1+\nu) \mu}{\pi \rho c} \int_{0}^{t} \int_{A\left(t_{1}\right)} \frac{V\left(t_{1}\right) p\left(x_{1}, t_{1}\right)}{\left\{k\left(t-t_{1}\right)\right\}^{1 / 2}} \phi_{1}\left(R_{1}\right) \mathrm{d} x_{1} \mathrm{~d} t_{1} \\
= & D-\frac{x^{2}}{2 r} \quad x \in A(t)
\end{aligned}
$$

where

$R_{1}^{2}=\frac{\left(x-x_{1}\right)^{2}}{4 k\left(t-t_{1}\right)}$

$E$ is Young's modulus, $\mu$ is the coefficient of friction and $V$ is the sliding speed. The first integral in eqn. (5) gives the elastic displacements caused by the pressure, the second defines the thermoelastic displacements, accumulated over the period of time $t$, and the right-hand side represents the initial curvature of the body (radius $r$ ) and includes an unknown rigid body displacement $D$.

The integrals should be performed over the domain $A(t)$ for which the two solids are in contact and this is also the range of definition of the integral equation. It is not known a priori, but it is determined by the conditions

$$
\begin{array}{ll}
p(x, t)=-\sigma_{y y}(x, 0, t) \geqslant 0 & x \in A(t) \\
u_{y}(x, t) \geqslant D-\frac{x^{2}}{2 r} & x \in \bar{A}(t)
\end{array}
$$

and

$$
\int_{A(t)} p(x, t) \mathrm{d} x=P(t)
$$

where $\bar{A}(t)$ is that part of the surface which is not in contact and $P(t)$ is the prescribed force pressing the bodies together. The two inequalities (7) and (8) state respectively that the contact tractions should be non-tensile and that there should be no interpenetration of material outside the contact area.

Once eqn. (5) is solved for $p(x, t)$, the surface temperature can be found from the equation

$$
T(x, t)=\frac{\mu}{2 \pi k p c} \int_{0}^{t} \int_{A\left(t_{1}\right)} \frac{V p\left(x_{1}, t_{1}\right) \exp \left(-R_{1}^{2}\right) \mathrm{d} x_{1} \mathrm{~d} t_{1}}{t-t_{1}}
$$




\section{Non-dimensional formulation}

For constant load $P$ and speed $V$, the number of parameters can be drastically reduced (to one) by introducing the non-dimensional variables

$x^{*}=x / A_{0}$

$A^{*}=A / A_{0}$

$T^{*}=3 \pi k \rho c T / 4 \mu P V$

$p^{*}=p A_{0} / P$

$t^{*}=k t / A_{0}^{2}$

where $A$ is the half-width of the area of contact and $A_{0}$ is the value of $A$ in the steady state and is given by [7]

$A_{0}=\frac{3 \pi(1-\nu) k \rho c}{4 \alpha \mu V E}$

Introducing eqns. (11) - (15) into eqns. (5) and (10) yields

$$
\begin{aligned}
& \frac{2}{\pi} \int_{-A^{*}\left(t^{*}\right)}^{A^{*}\left(t^{*}\right)} p^{*}\left(x_{1}{ }^{*}\right) \log \left|x^{*}-x_{1}{ }^{*}\right| \mathrm{d} x_{1}{ }^{*}- \\
& -\frac{3}{4} \int_{0}^{t^{*}} \int_{-A^{*}\left(t^{*}\right)}^{A^{*}\left(t^{*}\right)} \frac{p^{*}\left(x_{1}{ }^{*}, t_{1}{ }^{*}\right) \phi_{1}\left(R_{1}\right) \mathrm{d} x_{1}{ }^{*} \mathrm{~d} t_{1}{ }^{*}}{\left(t^{*}-t_{1}\right)^{1 / 2}} \\
& =\frac{2}{\pi}\left[-x^{* 2}\left\{\frac{A_{0}}{A(0)}\right\}^{2}+D^{*}\right]-A^{*}<x^{*}<A^{*}
\end{aligned}
$$

$T^{*}=\frac{3}{8} \int_{0}^{t^{*}} \int_{-A^{*}\left(t^{*}\right)}^{A^{*}\left(t^{*}\right)} \frac{p^{*} \exp \left(-R_{1}{ }^{2}\right) \mathrm{d} x_{1}{ }^{*} \mathrm{~d} t_{1}{ }^{*}}{t^{*}-t_{1}{ }^{*}}$

where $D^{*}$ is the dimensionless form of the arbitrary constant $D$ and $A(0)$ is the half-width of the contact area at $t=0$ given by

$\{A(0)\}^{2}=\frac{4 P\left(1-\nu^{2}\right) r}{\pi E}$

For the range of the integrals in these equations it is implicitly assumed that the contact area does not bifurcate. We can see from eqn. (17) that the solution depends on the single parameter $A^{*}(0)$.

\section{Discretization and numerical procedure}

The contact problem can be discretized in time and space by dividing the contact area into several strips of width $\Delta x$, replacing the actual 
pressure and heat input by piecewise-constant presentation and proceeding in increments of time. Computing the pressure and the temperature for a current time step we keep the heat input from the previous time step unchanged. This corresponds to the explicit scheme in the finite element method. However, an essential difference is that we deal with the surface rather than with the volume of the body. The influence of the uniform heat input distributed over a strip $\Delta x$ on the displacements and temperature at point $x$ can be conveniently expressed in terms of the solutions given in ref. 15 .

These results can be used to replace eqn. (17) for a current time step with a sum, expressed in terms of unknown pressures $p\left(x_{i}\right)(i=2,3, \ldots, N-1)$ at all interior points of the area of contact and the constant $D$. The set of algebraic equations is obtained by prescribing in turn $x=x_{i}(i=2,3, \ldots$, $N-1$ ). An additional equation is obtained by the discretization of eqn. (9). The non-linearity, which results from the fact that the area of contact is not known in advance, can be handled by trials and checks of inequalities (7) and (8). For a symmetric problem with a simply connected area of contact (which is the case for the examples treated below) the search can be simplified. We drop eqn. (9) and enforce eqn. (17) at the boundary point instead. Thus, we impose two conditions on this point. First, we require the pressure to be equal to zero (by dropping it from the unknowns); secondly, we force this point to be in contact. These two conditions are equivalent to requiring continuity of contact pressure at the boundary and this is equivalent to the inequalities (7) and (8) for simply connected contact areas. If the total force $F=\Sigma p_{i} \Delta x_{i}$ turns out to be, say, larger than $P$ so that eqn. (9) is violated, we release one point and repeat the procedure until a sufficiently accurate solution is obtained. In fact, we start the search with the area of contact found in the previous time step. Since the increment of time is always small, no more than one or two points are candidates for release, and non-linearity does not cause problems.

The attempt to use the piecewise-constant representation was not successful. The solutions were unstable for any reasonable number of space steps for $A(0)>5$. The pressures obtained differed significantly in value and sometimes in sign at neighboring points and the results did not converge with increasing numbers of points. Since the oscillations occurred on the scale of the discretization, the numerical procedure (not the process) should be blamed for the instability. The numerical procedure was stabilized when the piecewise-constant distribution was replaced with piecewise-linear representation of the heat input and the pressure. It can be constructed as a linear combination of triangular heat inputs (Fig. 2(a)). The same idea was used in ref. 17 for isothermal rolling of dissimilar elastic cylinders. The triangular heat inputs, in turn, can be obtained as a linear combination of linearly distribuled heal inputs (Fig. 2(b)). The displacements $u_{y}$ caused by a linearly distributed heat input $q_{1} x$ can be obtained by integration of the Green's function for a continuous point source in space giving 


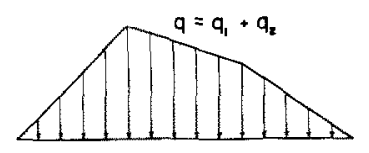

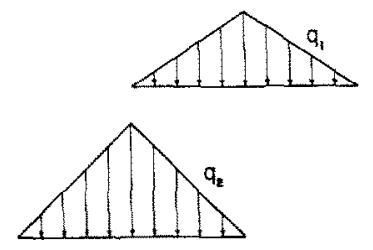

(a)

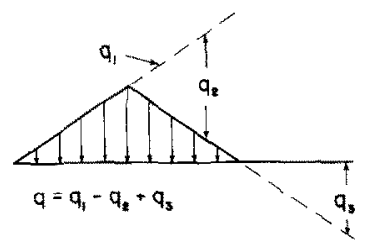

(b)

Fig. 2. The piecewise-linear representation of the pressure.

$$
\begin{aligned}
u_{y} & =-\frac{\alpha q_{1}(1+\nu)}{\pi \rho c k^{1 / 2}} \int_{0}^{t} \frac{\mathrm{d} t_{1}}{\left(t-t_{1}\right)^{1 / 2}} \int_{0}^{x} x_{1} \phi_{1}\left(R_{1}\right) \mathrm{d} x_{1} \\
& \equiv-\frac{q_{1} \alpha(1+\nu)(k t)^{3 / 2}}{\pi k \rho c} \phi_{2}(X)
\end{aligned}
$$

Numerically efficient series for $\phi_{2}(X)$ can be obtained from those given in ref. 15 by integration and are for small and large $X$ respectively

$\phi_{2}(X)=\frac{2 \pi x^{3}}{3}-\frac{4}{\pi^{1 / 2}} \sum_{i=0}^{\infty} \frac{\left(-2 X^{2}\right)^{i+1}}{(i+1)\left(4 i^{2}-1\right)(2 i+1) ! !}$

and

$$
\begin{aligned}
\phi_{2}(X)= & \frac{8}{3 \pi^{1 / 2}}\left(\frac{C}{2}+\log 2+\frac{4}{3}+\log X\right)-2 \pi X- \\
& -\frac{4}{\pi^{1 / 2}} \sum_{i=1}^{N} \frac{(2 i-1) ! !}{i\left(2 X^{2}\right)^{i}(2 i+3)(2 i+1)}
\end{aligned}
$$

where $C=0.577216 \ldots$.

The domains of acceptable accuracy of these series overlap at the value $X=2.0$.

The piecewise-linear representation produced physically meaningful results which are discussed in Section 6.

\section{Numerical results}

The method described was used to obtain results for a range of values of $A^{*}(0)$ between 3 and 500. The most important physical quantities of the process, the contact pressure and the surface temperature, were found as 


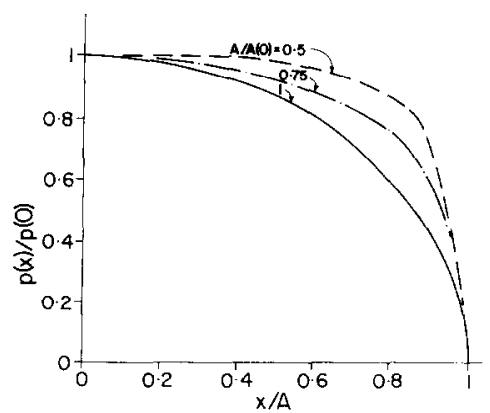

Fig. 3. Development of pressure distribution for $A^{*}(0)=20$.
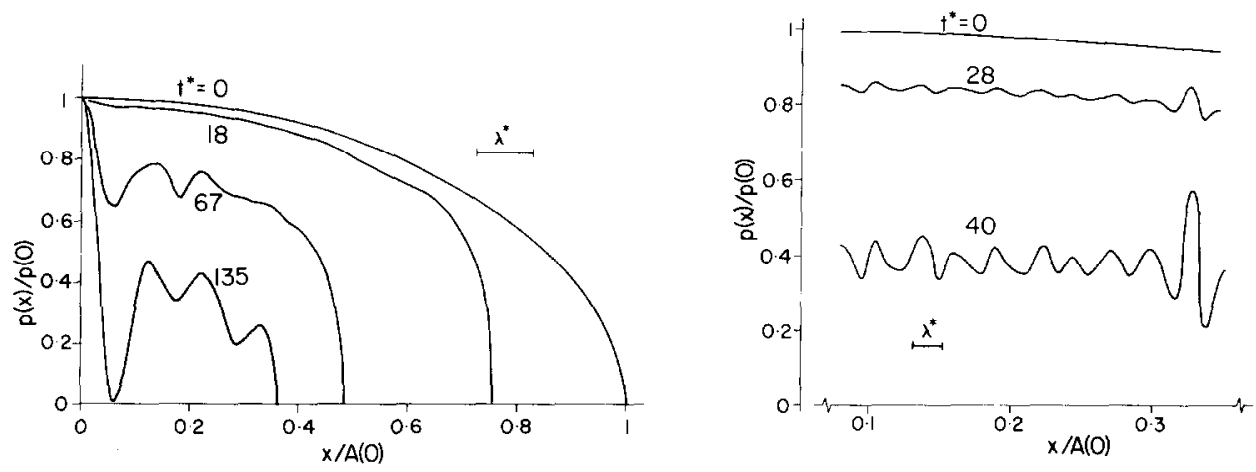

Fig. 4. Development of pressure distribution for $A^{*}(0)=100$. The wavelength corresponding to the maximum exponential growth rate $\sigma^{*}$ (see Fig. 8 ) is shown for comparison.

Fig. 5. Development of pressure distribution for $A^{*}(0)=500$.

functions of time. Some general conclusions about the nature of the process can be made. For $A^{*}(0) \leqslant 20$ the pressure changes smoothly from the initial (hertzian) distribution toward the steady state distribution (Fig. 3). For $A^{*}(0)=50$ some waviness in the pressure distribution was observed, but it was inconclusive whether it represented a numerical "noise" or a physical process. However, bifurcation did not occur. For $A^{*}(0) \geqslant 80$ a wavy perturbation develops (Figs. 4 and 5 ) and eventually the area of contact bifurcates. The wavelength of the perturbation is large compared with the scale of the discretization and the results converge with increasing numbers of points, showing that this is a real feature of the solution. The larger is $A^{*}(0)$, the earlier this happens. For $A^{*}(0)=80$ the area of contact bifurcates at $t^{*}=$ 170 while with $A^{*}(0)=500$ it bifurcates at $t^{*}=40$. Rates of shrinking of $A^{*}$ for some $A^{*}(0)$ are given in Fig. 6 (full curves). The full curves in Fig. 7 show dimensionless temperature at the midpoint of the contact area.

\section{Comparison with the simplified model}

It is interesting to compare these results with those obtained with the simplified model [10] in which the distorted body is approximated by a 

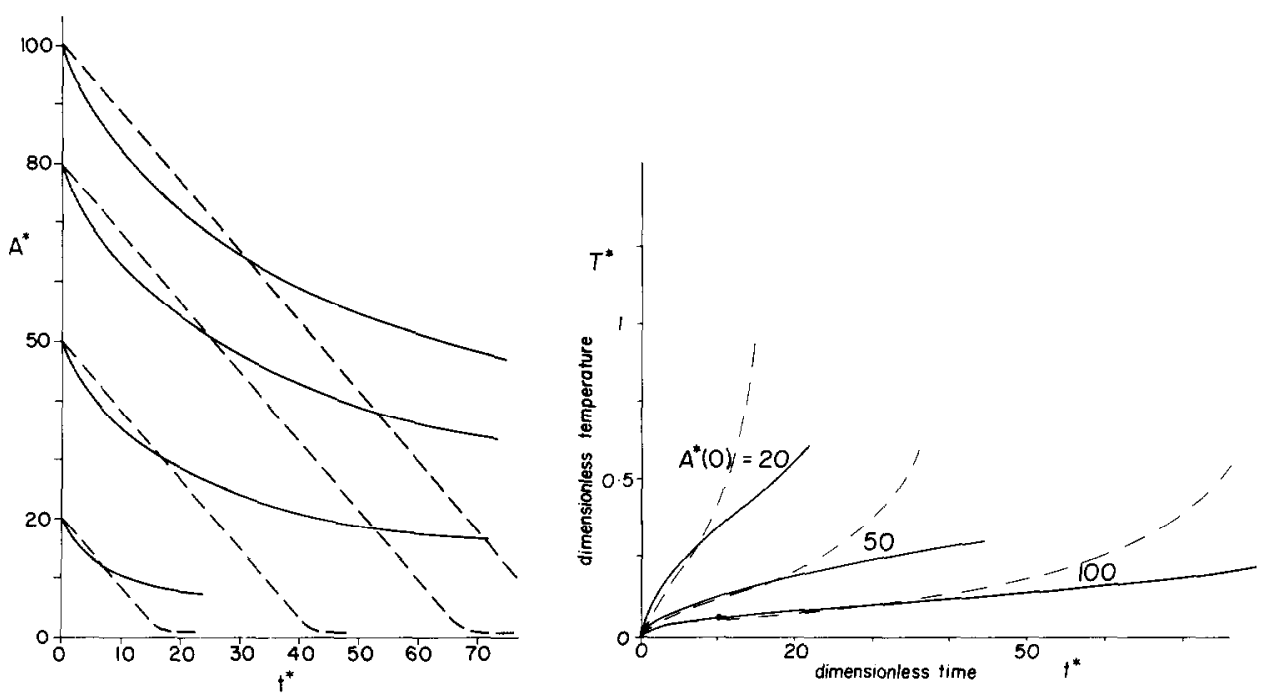

Fig. 6. Reduction of dimensionless contact half-width $A^{*}$ with time (-). Results obtained from the hertzian approximation (-- ) (Appendix A) are shown for comparison.

Fig. 7. Dimensionless maximum surface temperature $T^{*}$ as a function of time $t^{*}$ for $A^{*}(0)=20,50$ and 100 : - exact solution;---, hertzian approximation.

quadratic surface. The analysis given in ref. 10 relates to the axisymmetric problem, but the two-dimensional problem can be treated in the same way (see Appendix A). For constant velocity the governing equation has the form

$$
\begin{aligned}
& \frac{1}{\left\{A^{*}\left(t^{*}\right)\right\}^{2}}-\frac{1}{\left\{A^{*}(0)\right\}^{2}} \\
& =3 \pi^{1 / 2} \int_{0}^{t^{*}} \frac{\mathrm{d} t_{1}{ }^{*}}{\left\{A^{*}\left(t_{1}{ }^{*}\right)\right\}^{3}} \int_{0}^{A^{*}\left(t_{1}^{*}\right) / 2\left(t^{*}-t_{1}\right)^{1 / 2}} s^{2} \exp \left(-s^{2}\right) \mathrm{d} s
\end{aligned}
$$

When $t^{*}$ is small, this equation has the approximate solution

$$
A^{*}\left(t^{*}\right)=A^{*}(0)-\frac{3}{8} \pi t^{*}
$$

A direct numerical solution to eqn. (23) does not differ significantly from the straight line approximation until $A^{*}(t)$ falls below 3 (see the broken curves in Fig. 6). Corresponding curves for the maximum temperatures predicted by the simplified model are shown by broken lines in Fig. 7 . These two figures show that the simplified model gives acceptable results for temperature and order of magnitude results for contact area during the earlier stage of the process, but the error becomes unacceptable after the contact area has shrunk to about $60 \%$ of $A^{*}(0)$. We note that as the process develops, the simplified model overestimates the maximum temperature reached. 


\section{Comparison with Dow and Burton's solution}

As mentioned in Section 1, Dow and Burton [6] have shown that a sinusoidal perturbation in contact pressure distribution between two completely conforming sliding half-planes can grow if the sliding speed exceeds a certain critical value which depends on the wavelength. The situation in the present problem is different, in that the bodies make contact over a discrete area, but the waviness in pressure distribution observed in Figs. 4 and 5 suggests that some similar mechanism is involved and invites comparison with the unstable wavelengths found in ref. 6.

Dow and Burton's analysis (after adjustment of constants to the plane strain problem) shows that a contact pressure perturbation $\Delta p=p_{0} \sin (\omega x)$ will grow with time according to the equation

$\Delta p=p_{0} \exp (\sigma t) \sin (\omega x)$

where the exponential growth rate $\sigma$ is related to $\omega$ by the equation

$\left(\frac{\sigma}{k}+\omega^{2}\right)^{1 / 2}=\frac{\mu V \alpha E}{K(1-\nu)} \frac{k \omega}{\sigma}\left\{\left(\frac{\sigma}{k}+\omega^{2}\right)^{1 / 2}-\omega\right\}$

(see ref. 6, eqn. (9)). This equation can be simplified by introducing the dimensionless parameters

$\omega^{*}=A_{0} \omega \quad \sigma^{*}=\sigma A_{0}{ }^{2} / k$

and using eqn. (16) to give

$\left(\sigma^{*}+\omega^{* 2}\right)^{1 / 2}=\frac{3 \pi}{4} \frac{\omega^{*}}{\sigma^{*}}\left\{\left(\sigma^{*}+\omega^{* 2}\right)^{1 / 2}-\omega^{*}\right\}$

It should be noted that all physical parameters have disappeared from the equation. The solution of eqn. (27) is shown graphically in Fig. 8. The domain with negative $\sigma^{*}$ means that the waves with corresponding frequency $\omega^{*}$ decay. In the domain of positive $\sigma^{*}$ there is a particular wave frequency $\left(\omega^{*}=0.6\right)$ for which the rate of growth is the largest $\left(\sigma^{*}=0.5\right)$. This perturbation eventually dominates the process regardless of the initial amplitude of the corresponding wave. The corresponding wavelength

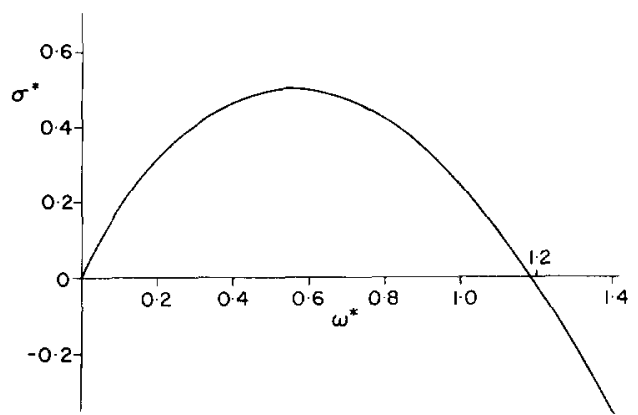

Fig. 8. Exponential growth rate $\sigma^{*}$ as a function of wave frequency $\omega^{*}$. 
$\lambda^{*}=\frac{2 \pi}{\omega^{*}}=10.5$

is shown in Figs. 4 and 5. We note that it compares well with the waviness obtained from the numerical results.

\section{Conclusion}

The numerical technique described enables us to follow the transient behavior of the system until it approaches the steady state for $A(0)<80$ and up to the onset of bifurcation for larger values. We must presume that after bifurcation occurs, the distribution of load $P$ between the several contact areas will change until eventually one area carries the whole load, after which approach to the steady state solution will be rapid. However, the present method is not suited to pursuing this phase of the process for two reasons as follows.

(1) While the use of Green's functions is very efficient for the earlier time steps, since it reduces the dimension of the problem, it becomes less efficient after many steps because at each step the accumulated thermal distortions of previous steps have to be re-computed. This problem is compounded by the fact that, when bifurcation occurs, a larger number of spatial points are needed to give an adequate representation of the pressure distribution.

(2) The iterative method used for the non-linear contact problem, prescribing the contact area and iterating on the total load $P$, will not work when the contact area bifurcates, since there are now several contact boundaries to find.

Problem (2) could be overcome by developing a different iterative algorithm but, in view of (1), we feel that it is more appropriate to use a volume rather than a surface representation to describe this phase of the process. Work on such a representation is still in progress and will be reported later.

\section{Acknowledgment}

The authors gratefully acknowledge support from the U.S. Army under Contract DAAG 29-82-K-0001.

\section{References}

1 R. C. Parker and P. R. Marshall, The measurement of the temperature of sliding surfaces, with particular reference to railway blocks, Proc., Inst. Mech. Eng., London, 158 (1948) $207 \cdot 229$. 
2 J. R. Barber, The influence of thermal expansion on the friction and wear process, Wear, 10 (1967) $155-159$.

3 J. R. Barber, Thermoelastic instabilities in the sliding of conforming solids, Proc. $R$. Soc. London, Ser. A, 312 (1969) 381 - 394.

4 R. A. Burton, V. Nerlikar and S. R. Kilaparti, Thermoelastic instability in a seal-like configuration, Wear, 24 (1973) $177-188$.

5 R. A. Burton and V. Nerlikar, Large disturbance solution for initially flat frictionally heated thermoelastically deformed surfaces, J. Lubr. Technol., 97 (1975) $546-551$.

6 Th. A. Dow and R. A. Burton, Thermoelastic instability of sliding contact in the absence of wear, Wear, 19 (1972) $315-328$.

7 J. R. Barber, Some thermoelastic contact problems involving frictional heating, Q. J. Mech. Appl. Math., 29 (1976) 1 - 13.

8 R. A. Burton, S. R. Kilaparti and V. Nerlikar, A limiting stationary configuration with partially contacting surfaces, Wear, 24 (1973) 199 - 206.

9 R. A. Burton and V. Nerlikar, Effect of initial surface curvature on frictionally excited thermoelastic phenomena, Wear, 27 (1974) $195-207$.

$10 \mathrm{~J}$. R. Barber, The transient thermoelastic contact of a sphere sliding on a plane, Wear, $59(1980) 21$ - 29 .

11 J. R. Barber, T. W. Beamond, J. R. Waring and C. Pritchard, Implications of thermoelastic instability for the design of brakes, $J$. Tribol., to be published.

12 J. Dundurs, J. Appl. Mech., (September 1969) 650 - 652, Discussion.

13 J. R. Barber, Distortion of the semi-infinite solid due to transient surface heating, Int. J. Mech. Sci., 14 (1972) 377 - 393.

14 H. S. Carslaw and J. C. Jaeger, Conduction of Heat in Solids, Clarendon, Oxford, 2nd edn., 1959 , p. 258.

15 J. R. Barber and C. J. Martin-Moran, Green's functions for transient thermoelastic contact problems for the half-plane, Wear, 79 (1982) $11-19$.

16 W. L. Miller and A. R. Gordon, Numerical evaluation of infinite series and integrals, J. Phys. Chem., 35 (1931) $2785-2884$.

17 R. H. Bentall and K. L. Johnson, Slip in the rolling contact of dissimilar rollers, Int. J. Mech. Sci., 9 (1967) 389 - 404.

\section{Appendix A}

\section{A.1. Simplified model}

In ref. A1 an approximate solution was developed based on the representation of the thermally deformed body by a quadratic surface:

$u_{y}(x, t)=C_{0}(t)+C_{1}(t) x^{2}$

We present here the corresponding results for the two-dimensional problem for completeness. From eqn. (A1) it follows that the pressure distribution is hertzian at all times and hence

$p(x, t)=\frac{C_{1}(t) E}{1-v^{2}}\left[\{A(t)\}^{2}-x^{2}\right]^{1 / 2}$

where the half-width of the strip is given by

$A(t)=\frac{P\left(1-\nu^{2}\right)}{2 \pi C_{1} E}$ 
The heat input

$$
\begin{aligned}
q(x) & =\mu V p(x) \\
& =\frac{C_{1} E \mu V\left[\{A(t)\}^{2}-x^{2}\right]^{1 / 2}}{1-\nu^{2}}
\end{aligned}
$$

and hence the thermoelastic displacements are

$u_{y}(x, t)=-\frac{\alpha E \mu V}{k^{1 / 2} \pi \rho c(1-\nu)} \int_{0}^{t} \int_{-A\left(t_{1}\right)}^{A\left(t_{1}\right)} C_{1}\left(t_{1}\right) \frac{\left[\left\{A\left(t_{1}\right)\right\}^{2}-x_{1}{ }^{2}\right]^{1 / 2} \phi_{1}\left(R_{1}\right) \mathrm{d} x_{1} \mathrm{~d} t_{1}}{\left(t-t_{1}\right)^{1 / 2}}$

from eqn. (3). Introducing eqn. (A1) into the left-hand side of eqn. (A5) and separating the terms with the factor $x^{2}$, we obtain in the same way as in ref. A1

$$
C_{1}(t)=\frac{4 E \alpha \mu V}{\pi^{1 / 2} \rho c(1-\nu)} \int_{0}^{t} \frac{C_{1}\left(t_{1}\right)}{A\left(t_{1}\right)} \int_{0}^{D} s^{2} \exp \left(-s^{2}\right) \mathrm{d} s
$$

where

$D=\frac{A\left(t_{1}\right)}{2\left\{k\left(t-t_{1}\right)\right\}^{1 / 2}}=\frac{A^{*}\left(t_{1}{ }^{*}\right)}{2\left(t^{*}-t_{1}{ }^{*}\right)^{1 / 2}}$

After replacing $C_{1}$ from eqn. (A3), the dimensionless form of eqn. (A6) becomes

$$
\frac{1}{\left\{A^{*}\left(t^{*}\right)\right\}^{2}}-\frac{1}{\left\{A^{*}(0)\right\}^{2}}=3 \pi^{1 / 2} \int_{0}^{t^{*}} \frac{\mathrm{d} t_{1}{ }^{*}}{\left\{A^{*}\left(t_{1}{ }^{*}\right)\right\}^{3}} \int_{0}^{D} s^{2} \exp \left(-s^{2}\right) \mathrm{d} s
$$

For large $D$ (short time solution)

$\int_{0}^{D} s^{2} \exp \left(-s^{2}\right) \mathrm{d} s \approx \frac{\pi}{4}$

and we obtain the straight line approximation

$A^{*}\left(t^{*}\right)=A^{*}(0)-\frac{3 \pi}{8} t^{*}$

As the dimensionless time $t^{*}$ tends to infinity, eqn. (A8) predicts that $A^{*}\left(t^{*}\right)$ tends to $8 / 3 \pi$ instead of unity. This gives some measure of the accuracy of the approximation.

Reference for Appendix A

A1 J. R. Barber, The transient thermoelastic contact of a sphere sliding on a plane, Wear, 59 (1980) 21 - 29. 